\title{
Breaking the Rules of Direct Manipulation
}

\author{
David Golightly \& David J. Gilmore \\ Psychology Dept \\ University of Nottingham \\ Nottingham, NG7 2RD, UK \\ dag@psyc.nott.ac.uk
}

\begin{abstract}
There is a clear conflict between making an interface easier to use and making computer-based problems easier to solve. Making the control of the problem-solving domain more complex helps uscrs to reach their solutions in less steps. Previous explanations of this phenomena have been centred in how the interface impacts on learning or planning of the problem solving domain. This paper adds a new element to this work by drawing on observational analysis. Interface differences are closely related to the types of strategy a user employs. A more complex interface makes the user more sensitive to their problem solving situation. Explanations of the phenomena are reconsidered in the light of strategy analysis. The effect occurs in problem solving interfaces because the domain specific requirements of the interface cannot be divorced from the domain independent goals. The application of the effect to design is discussed in relation to several areas.
\end{abstract}

Keywords: Interaction design, task analysis, problem solving, cognitive load, direct manipulation.

\section{INTRODUCTION}

The interface is the access point to the computer. If the computer contains information we wish to manipulate, learn about and generally operate on in any way then we do so through the interface.

$\mathrm{HCI}$ is often a drive towards User Centred Design. Encompassed within this drive is the accepted notion that, if the interface is a window, it should not be opaque or distorted, but clear, smooth and seamless. This will allow manipulation of the data with the user almost unaware that they are communicating through the interface medium. This is exemplified in the Apple Interface Guidelines. Objects represented by the interface should be clearly understood by a user, should respond almost instantaneously to input with feedback and should represent as closely as possible the objects

Human-Computer Interaction: INTERACT'97 S. Howard, J. Hammond \& G. Lindgaard (editors)

Published by Chapman \& Hall OIFIP 1997 
they refer to in the actual data being manipulated. Also, a user should be allowed to quickly reverse actions with the most minimal damage to their overall state. These are some of the key principles behind Direct Manipulation (Norman, 1986).

The benefits of Direct Manipulation are the rapid learning of functionality for novices coupled with easeof-execution for experts with any given system. Implicit with these benefits is the idea that making an interface conform to Direct Manipulation reduces the mental workload necessary to operate any application. In other words, by making the interface neutral to the user they can concentrate less on interface goals and more on their domain goals.

Increasingly, the 'neutrality' of the interface is coming into question. This paper focuses on the concern that different tasks require qualitatively different interface requirements. Easier interaction is not always more effective interaction.

Many conclusions and guidelines about manipulation style have been drawn from the domains such as word processing or spreadsheet tasks. In such situations the domain knowledge is already known by the user. What is new to them is the interface. A group of studies have looked at how the interface affects domain learning (Svendsen(1991), O'Hara \& Payne (1994), Golightly, Gilmore \& Churchill (1996)) where the domain, as well as the interface, is new to a user. These studies deal specifically with problem solving tasks.

The Svendsen study looked at the Tower of Hanoi task. Both the O'Hara \& Payne and the Golightly et al task looked at sliding tile 8-puzzle (see figure 1). All three studies found that increasing the difficulty of manipulating these problem domains improved users performance. Making a move more difficult leads the user to take fewer moves to solve the puzzle.

Several explanations have been offered as to why this effect may occur. It may be the case that making control of the interface more difficult leads the user to develop a richer knowledge of the domain. This knowledge is explicit; it can be transferred from one task to another and is more easily open to verbal report. Svendsen argues that the standard direct manipulation interface promotes implicit learning. The knowledge is robust but is not open to conscious retrieval and adaptation. This makes it more difficult for the user to be aware of situations where knowledge about a domain is relevant.

For O'Hara and Payne, the more difficult interface leads the user to plan more. The cost of carrying out an action with a more difficult interface warrants the user to make a more efficient choice. In order to choose the optimum move the user may work several moves ahead mentally to assess its outcome. This enhanced planning is not necessary for a Direct Manipulation interface. In that situation it is much easier to perform trial-and-error action and asses the impact of a move by simply executing it.

What is clear from these studies is that an interface can have an influence on how a user performs in a domain. If the domain has a strong problem solving element then a more difficult interface will help the user to solve the problem more efficiently. The Golightly et al study found that only certain types of difficulty induced this improved performance. The execution had to be made cognitively more difficult. This was done by moving the control of the puzzle away from the puzzle itself and onto a separate set of buttons on the interface. This did not require any extra interface operations from the user to move a tile (though, as we shall see, it does involve extra cognitive activity). It is moving the point of input away from the point of action (and thus breaking one of the key rules of Direct Manipulation) that makes the difference.

These studies have all used gross measures of behaviour. They have taken dependent variables such as time per move, time per puzzle or number of errors and applied statistics to show the effect is valid. The results are then used to develop theory about what users are doing to exhibit these discrepancies between interfaces. This paper offers some observational analyses of the moves that users make in terms of their strategies, goals and subgoals. These observations clarify some of the explanations of the effect and point to where it will be of use in design.

Observing patterns of problem solving might be seen as a subjective process. There are two ways 10 give it rigour. The first is to use data collected from a conventional study. This can be analysed statistically to show it is a valid replication of the effect and 


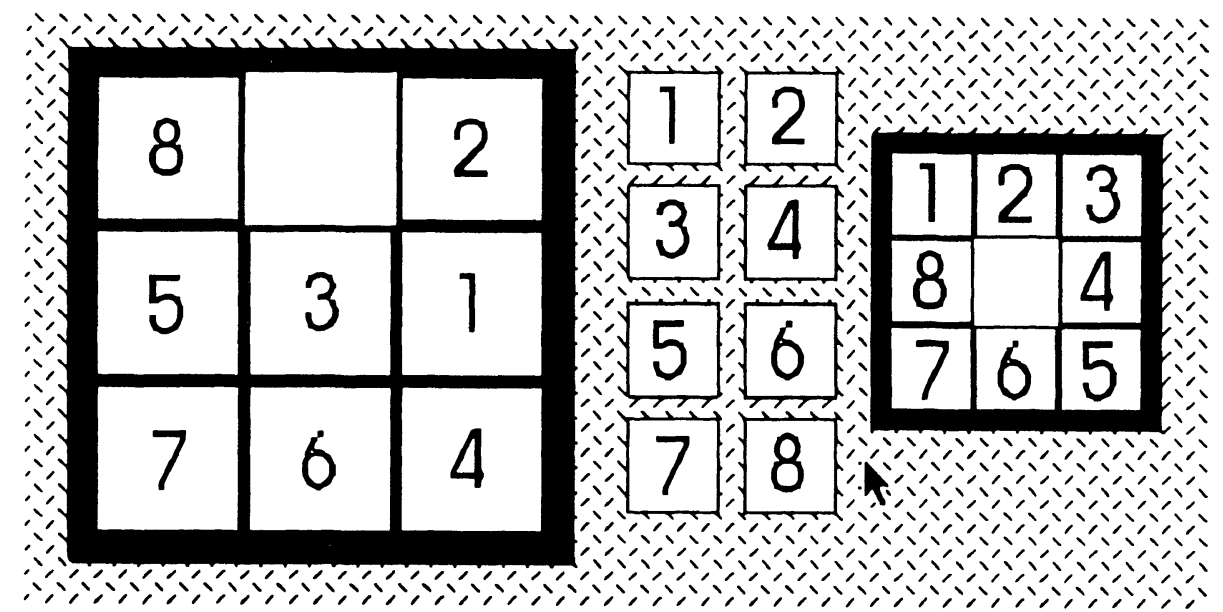

Figure 1. The IM interface. The actual puzzle is on the left, the goal state is shown on the right and the buttons used for moving the tiles ( hidden on the DM interface) are in the middle.

cligible for more qualitative analysis. The second is to use a candidate strategy. The candidate is formally defined beforehand and then used as a comparison to see in which ways any observed strategy conforms or differs. These two features strengthen any conclusions that are drawn from the observation.

\section{A STUDY}

\subsection{Method}

The following is a brief description of the experiment used to elicit results for further analysis. Two groups of participants were given a set of 8-puzzles with various starting configurations. The puzzles were presented on an Apple Macintosh. Participants were asked to complete as many as possible in 15 minutes. Ilalf of the participants were given an Indirect Manipulation (IM) interface (see figure. 1). In order to move a tile they had to click on the corresponding button in the middle of the display. The distance hetween the buttons and the display of the puzzles meant that it was not possible to see both at once. This was verified by the subject's observations after the experiment. For the DM interface, the participants had to click on the actual tile on the puzzle. For this interface the buttons were hidden.

Each group were given the same puzzles in the same order. The puzzles were ordered so that even those participants who only solved a few puzzles had both easy and harder starting configurations.

The following measures of performance were taken; average time to make a move (In transformed), number of puzzles solved in the 15 minutes and average number of moves to solution.

\subsection{Results}

The two groups differed in the number of puzzles they solved in 15 minutes. The IM group completed significantly less puzzles $(\mathrm{IM}=5.33$, $\mathrm{DM}=$ $8.25)(\mathrm{F}(1.22)=26.165, \mathrm{p}<0.01)$. They also took longer over their moves (mean IM move time $(\ln )=$ 0.46 ) than the DM group (mean DM move time $(\ln )=$ $-0.23)(\mathrm{F}:(1,22)=79.79, \mathrm{p}=\mathrm{p}<0.01)$.

The IM group took fewer moves to solve their puzzles with a mean of 75 moves as opposed to the DM groups $104.8(\mathrm{~F}(1.22)=11.64, \mathrm{p}=\mathrm{p}<0.01)$.

A further dependent variable was taken, standard deviation of move times (ln). Briefly, with planned action, a problem solver will spend a long time at one 
particular point in the problem. This is when the planning occurs. Then the next few moves will occur in quick succession as the plan is executed. There will then be another long period while the next few moves are planned. The standard deviation of move times is a numerical measure of the range of move times that a participant exhibits. A higher standard deviation correlates with greater planning. ${ }^{*}$ The IM group displayed a greater range of move times (SDev (In) for $\mathrm{IM}=0.65)$ than the DM group (SDev (In) for DM = $0.55)(\mathrm{F}(1,22)=5.96, \mathrm{p}=0.023)$.

\section{ANALYSING STRATEGIES 3.1 Methodology}
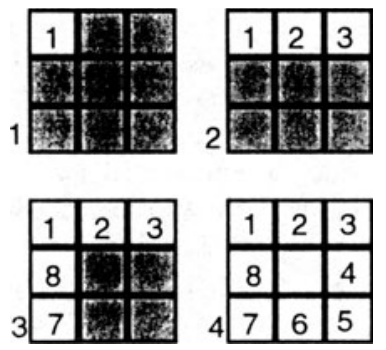

Figure 2. Subgoals and final goal state of the 8 puzzle when using the candidate 'Place' strategy (the greyed squares are not relevant to any given state.)

These results clearly replicate previous findings of Svendsen, O'Hara \& Payne and Golightly et al. The DM interface allowed for the participants to make quicker moves and, as a result. allowed for them to complete more puzzles in the allotted period. The IM interface allowed the participants to complete the puzzles in fewer moves. They also displayed a higher range of move times suggesting planning activity.

The two interfaces do lead to different performance. Is it possible to define this in the nature of the participant's problem solving? The IM interface is promoting more efficient problem solving in that they take fewer moves to solution. They are making less

* $A$ complete explanation of the theory behind this measure is offered in Golightly et al.

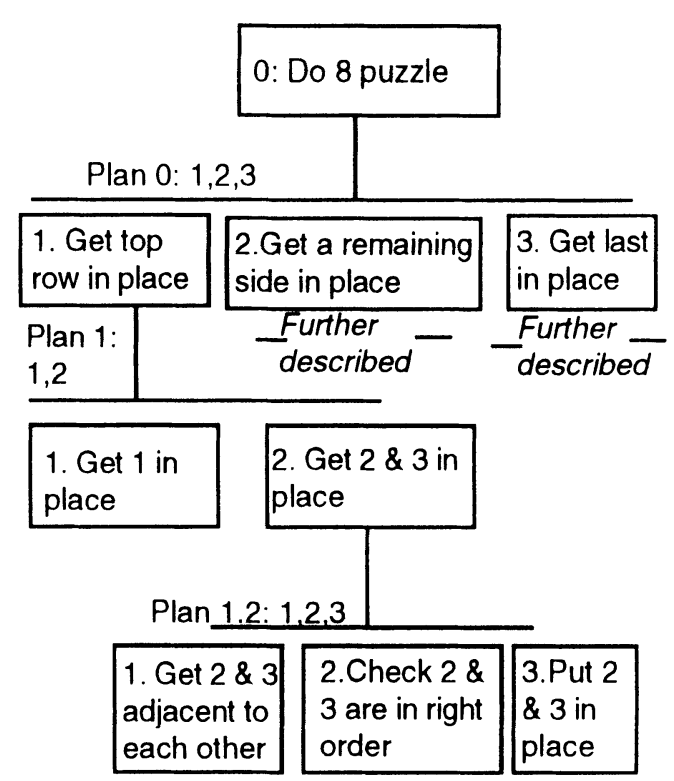

Figure 3. Heirarchical Task analysis of the candidate 'Place' strategy.

errors in their selection of which tile to move and making choices which direct them more quickly to the goal state. Using observation to explore the nature of the interface reveals it also promotes different moves to solution.

A candidate strategy was used as a comparison. One obvious strategy to employ can be described as a 'place' strategy. Here, the problem solving involves basic problem reduction. The aim of the strategy was to achieve a series of subgoals; first. place a corner then complete that side. A second side is then completed and finally the last 3 tiles are put in place (see figure 2.)

Though this is only a loose description of the strategy, further rigour can be added by describing it within a conventional formalism. In this case a Heirarchical Task Analysis (Shepherd, 1989) of the subgoal structure was used.

The IITA constrained the description of the strategy. By having a formalism of the subgoal structure it was possible to predict from the IITA which subgoal would be attempted next. However. it was not possible to start making predictions about the actual moves that would be made (e.g. helow the level 
of plan 1.2. The plans marked with '-further described-' had also been constructed at two more levels of complexity but no further). As a result, the analysis made no commitment to defining the moves taken to reach a subgoal.

A feature of having the constrained formalism was that it made clear how much a new strategy would deviate from the candidate strategy. Some participants could form the same subgoal structure but with other parts of the puzzle substituted in (e.g. tile 3 could have been placed first instead of tile 1). Some strategies would differ radically from the candidate to the point where they are qualitatively different. Alternatively it would be clear when no strategy was being used whatsoever.

The performances of the same 24 participants used for the statistical analysis were played back on a viewer application. Similarities and differences between the candidate strategy and the actual strategy were noted. Though problems of subjectivity have been discussed the strategic features of the two groups were striking enough to justify this approach.

Of the $12 \mathrm{DM}$ participants 11 used either the candidate strategy or a derivative. These derivations involved slight substitutions e.g. doing the left-hand row instead of the top row. However the subgoal structure was in essence the same. What was clear was that the subgoals were solved in a trial-and-error manner sometimes taking hundreds on moves to complete a side or to position the remaining five tiles.

The difficulty in perceiving any structure to the solution of the subgoals was in line with the HTA. It was difficult to predict a very deep hierarchy of sub goals in the task analysis but it appears that DM participants do not hold a deep hierarchy of subgoals anyway. They appeared to be focusing on one quite high level subgoal and using trial-and-error performance to achieve it. Within participants the strategies were regimented; participants found the strategy within one or two moves and stuck with it.

The IM group was much more loose in their use of strategies. They seemed to be far less consistent in their choice of strategy. There was a strong tendency to get the tiles in the right order first before trying to position them. The candidate strategy might be used on one puzzle but not on the next. Quite often it was hard to see any deliberate strategy. In essence, the IM participants appeared much more sensitive to the particular state the problem was in at any given point. They were able to make choices about which move to make which that more relevant to the position they were in rather than attempting to follow a rigid structure. It is important to remember that both groups were given the same puzzles in the same order. So, it is not the case that each groups strategy selection was a result of the puzzles they were presented with.

\section{INTERPRETATION}

The problem solving of the DM group appears rigid to the IM groups fluid, state-sensitive problem solving. These strategic observations give extra weight to explanations offered by O'Hara \& Payne and Svendsen, these being a) that the more complex interface improves planning b) that the more complex interface leads to richer, more explicit learning. We do not see these two explanations as conflicting. It is more likely the case that extra planning leads to more explicit understanding of the problem domain later on. The interpretation of the observational analysis makes this link.

We take the DM case first. When the problem solving can take place at the point of action the user does not need to build a deep cognitive representation of the problem. This is because the action can be based at the display which represents the problem for the user. A goal or sub goal can be held and the user can just try to get the display to match it using trial-anderror. This would explain the type of behaviour that the DM group exhibited.

If the point of action is moved away from the point of consequence then the user cannot use the display based representation. In order to reduce the effort of switching from the two points the user huilds a cognitive representation of a small part of the problem state. They use the representation at the point of interaction (the buttons) for a few moves before checking against the actual puzzle display.

This would explain why the IM group show a higher range of move times. On some moves the representation will be being built from the display. Also there will be moves where the internal and external representations must be checked against each 
other. But on other moves the representation will already be internalised so the cognitive effort for these moves will be much less. On these moves the response times will be lower.

Building an internal representation explains the more fluid problem solving. The DM user needs only to focus on the particular tile to be moved (e.g. focusing on ' 1 ' to get it in the corner). The IM user has to internalise the surrounding tiles as well to build this representation. So, they become sensitive to more of the problem state. This results in the strategic behaviour that the IM group shows.

This broadly conforms to the O'Hara \& Payne explanation of the effect. (The IM interface is not promoting planning in the strictest sense of the word. But it does promote the building and manipulation of an internal representation. However, it could be argued that the action is not planned but situated, albeit in an internal representation; this has been covered by Vera \& Simon (1993). We acknowledge this debate but do not wish to make a commitment to either viewpoint at this time.)

Having to build this internal representation requires dceper processing of the problem domain. This would, in turn. lead to the learning benefits that have been predicted with a more difficult problem solving interface.

\section{ANALYSING THE INTERFACE}

So far, we have defined the differences in performance between the two interfaces. But the discussion has been based in the differences of problem solving style. There must be an element to interfaces which creates these effects in the problem solving case. What is it about the problem solving situation that makes it a special case of interaction?

Using task analysis of the two interfaces defines how they are different and how this relates to the problem solving behaviour we have described. Both interfaces have identical grammatical consistency. Both have an equivalent representation of the problem domain. What differs between them is the set of procedures for manipulating a tile i.e. the unit task (Card, Moran \& Newell, 1983).

For the purposes of this discussion the analysis will be anchored in the N-GOMS-L notation (Kieras,
1989). This notation has a parsimony which is all that the current analysis requires. Also, his discussion draws a line between device specific goals, particular to the application that is being used, and device independent goals which are part of the domain that is being worked on.

This helps us to define the types of goal a user has to achieve in this problem solving case. One level of device independent goal in the 8-puzzle is to complete an 8-puzzle. The next level of device independent goal is moving a tile. That is, solving a puzzle and moving a tile are not related to the interface that is being used. They are general to the domain of solving 8-puzzles. What is specific to the interface is the methods for achieving that goal.

The action of moving a tile in the DM interface consists 3 steps.

Method 1. Accomplishing goal of move tile (DM)

Step 1. Select tile

Step 2. Move cursor to tile

Step 3. Click mouse

Moving with the IM interface is almost identical except for step 2 .

Method 2. Accomplishing goal of move tile (IM)

Step 1. Select tile

Step 2. Locate corresponding button

Step 3. Move cursor to button

Step 4. Click mouse

The selection (step 1) in these analyses is not a selection of a method, as is normally the case in a GOMS type analysis. It is a marker under N-GOMS-I . notation for a domain independent activity or a 'complex cognitive process' (Keiras, 1989, p.140) that is off-line from the analyses. The 'select tile' marker denotes the cognitive activity which is the domain related problem solving. It is at this point that the type of subgoaling we have observed occurs.

The domain specific component of these interfaces is the method for moving the tile once it has been selected (DM step $2 \& 3$; IM step 2, 3, 4). The difference between the two methods clearly illuminates the difference between the two interaction styles. The 
IM method has the extra action of locating the button. The types of strategic differences we have described and the explanations for them are driven by users trying to minimise the effect of this extra step. (NB. Though there is an extra action it is still part of the overall operation of moving a tile. It is this increased complexity that forces the performance difference, not simply adding an extra operation of very low complexity as in the Golightly et al study. Such an extra operation can be easily executed by the user so that it does not induce any extra cognitive load. )

The centre of this effect is that a small difference results in a large in impact on step 1-selecting a tile. This is because it occurs in a problem solving interface. What is different about problem solving situations, as opposed to tasks such as text editing or spreadsheets, is that the off line marker 'select tile' is a vital part of the method.

The assumption with most task analysis methods is that expert performance exists both in carrying out the method and in carrying out the cognitive activity that the off-line markers encompass. If expert performance cannot be guaranteed for the domain independent cognition then it is presumed that this has occurred at some other point in time. So, in a GOMS analysis of word processing, it is assumed that the document is already marked up before the user starts text editing.

But how can domain related cognition be moved off-line in an interface that is designed to support online cognition? In the problem solving situation the domain independent cognition is an integral part of the task.

This can be summed up as follows. The method of operation is not neutral to a selection process when the selection of the item to be operated on, rather than the execution of that operation, is the purpose of the interaction. Not only is the method not neutral, but our evidence shows that making it more complex leads the user to make more on-target selections.

It is not necessary for all of the extra cognitive activity to temporally occur at step 1 . But at some point a user reflects more on the domain independent act of selecting a tile because the method of executing that selection has been made more complex.

\section{A PRINCIPLE FOR DESIGN}

We now have some clear ideas about how, why and where more difficult interfaces should help problem solving. The observational data gives us the 'how'- the more difficult interface makes problem solving more efficient by making it more fluid and sensitive to the current problem state. From this we have enhanced existing theories of 'why'- moving the point of action away from the point of consequence encourages the problem solver to develop their own representation of the domain. This leads to deeper learning of the problem domain. The 'where' comes from considering the relationship between the device specific cognition and the device independent cognition- the more complex interface can help the user to make selections when their domain independent goal is problem solving.

There have been many discussions of how traditional cognitive approaches can trade with more holistic, situational studies to build more effective interactions while, at the same time, enhancing psychological theory (e.g. Landauer, 1987). The situation we have here is an excellent opportunity for this trade.

There are many research questions that can be further explored in the laboratory. For instance, this paper has considered how the interface impacts on learning. Much more work needs to be done into understanding how robust these effects are over time and in the face of transfer to other tasks. The laboratory would be an ideal place to study the effects of training regimes. A study is currently being analysed to explore these aspects of the effect.

This is only one side of the laboratory/ real world relationship. The time has come to really consider whether more complex interaction can be of use in actual interfaces. We can say with certainty that it is not a general design principle. When most users approach an application they are already aware of what they intend on doing. The key issue for a user is normally not 'what do I want to do?' but 'how do I do it?' Here the interface should be the common conception of a transparent window. Making the task user centred in such cases means making the how to do it' knowledge quick to learn, quick to execute and as engaging as possible. 
But there is a second situation where making decisions about choosing what to do efficiently takes precedence over the ease of doing it. Consider safety critical systems. Infrequent, high-cost failures need additional contextual information (Johnson, 1995). A common occurrence in accident situations is that operators focus in on performing one subgoal. Their focus is such that they fail to see other factors which may be giving them additional or even conflicting information. In such situations, an interface which allows an operator to see a broader context and make decisions that are more sensitive to the overall problem state would be highly advantageous. This is exactly the sort of benefits a more complex interface could deliver.

These interfaces are harder to learn how to work. An operator would not want to be faced with a highly counterintuitive interface in a moment of crisis. The question of training users so that the interface does not completely throw them has to be addressed. Equally such interfaces do not make you any quicker at your task. If speed is an issue then a more complex interface will probably slow down the overall time taken to complete a task. But if the domain involves making as few decisions as possible and making them as accurately as possible then this type of interface may offer much.

The safety critical system is an extreme case. There are a whole other range of situations that may benefit, either in whole or in part, from an interface which supports problem solving. Direct manipulation interface in educational situations may not always be of benefit (Gilmore,1996). The direct manipulation interface is supposed to 'engage' the user. Maybe in a learning situation it engages the learner in the interface rather than the information he or she is manipulating. Making the interaction less direct would make a learner focus more on the domain they are working in.

Exploratory navigation, whether in hypertext environments or more literally in virtual environments, might be made more effective by interaction styles that force the user to be sensitive to the information they have available to them at any one time. There also may be smaller parts of larger applications where the problem solving interface may be appropriate. A user can be aware of the tasks they wish to perform but may occasionally come across sub tasks which they have make decisions about. At these points it may be advantageous to support this decision making process.

\section{References}

Card, S.K., Moran, P.T. \& Newell, A. (1983) The Psychology of Human-Computer Interaction. Lawrence Erlbaum Associates, inc.

Gilmore, D. J. (1996) The relevance of $\mathrm{HCl}$ guidelines for educational interfaces. in MachineMediated Learning, 5(2): 119-133.

Golightly, D., Gilmore, D. J. \& Churchill, E.F. (1996) Puzzling Interfaces: the relationship between manipulation and problem solving in Thimbleby, $\mathrm{H}$. \& Blandford, A. HCI '96: Adjunct proceedings

Johnson, C. W. (1995) Decision theory and safety critical interfaces in Nordby, $\mathrm{K}$, Helmerson, P.H. Gilmore, D. J. \& Arnesen, S. A. eds. Proceedings of Interact '95.

Kieras, D. (1988) Towards a practical GOMS model of methodology for user interface design. In Helander, M., ed Handbook of Human-Computer Interaction. Amsterdam: North-Holland.

Landauer, T. K. (1987) Relations between cognitive psychology and computer system design. in Carroll, J. M. Interfacing thought: cognitive aspects of Human-Computer Interaction. Cambridge, MA: MIT.

Norman, D.A. (1986) Cognitive Engineering. in Norman D.A. \& Draper S.W. User Centred System Design: New Perspectives in Human-Computer Interaction. Lawrence Erlbaum Associates, inc.

O'Hara, K \& Payne, S.J. (1994) Cost of Operation Affects Planfulness of Problem-Solving Behaviour. School of Psychology, University of Wales, College of Cardiff.

Shepherd, A. (1989) Analysis and training in information tasks. In Task Analysis for HumanComputer Interaction ( Diaper, D. ed.) Chichester: Ellis-Harwood.

Svendsen, G.B. (1991) Influences of Interface Style on Problem Solving. International Journal of ManMachine Studies, 35: 379-397.

Vera, A. H. \& Simon, H. A. (1993) situated action: a symbolic interpretation. in Cognitive Science $17,7-48$. 